\title{
SMART FLUID SELF ADAPTIVE DAMPER SYSTEM (SFSADS)
}

\author{
Santhosh Sivan. $\mathrm{K}^{1}$, Chandrasekar Sundaram ${ }^{2}$ and Hari Krishnan. $\mathrm{R}^{3}$ \\ ${ }^{1,2}$ Department of Automobile Engineering, \\ Anna University, MIT, Chennai, India \\ autosash@yahoo.com \\ chandys92@gmail.com \\ ${ }^{3}$ Department of Aerospace Engineering, \\ Anna University, MIT, Chennai, India \\ rharikrish93@gmail.com
}

\begin{abstract}
This paper is on fluid damper systems, which is self-adaptive and used to increase the speed and acceleration of vehicles. In normal dampers there is a time lag leading to break in contact between the wheel and the road reducing the acceleration. Now, our idea is about making the suspension adapt to varying road conditions. Due to irregularities in asphalt, a change occurs in the suspension damper position with the aid of the magnetic field and causes the suspension to change from softer to harder and vice versa giving more acceleration and increasing the pace of the vehicle.
\end{abstract}

\section{KEYWORDS}

Fluid Damper, Self-Adaptive, Speed, Acceleration, Suspension, Magnetic Field, Softer \& Harder

NOMENCLATURE

$\begin{array}{lll}W & - & \text { Load acting on a spring }(\mathrm{N}) \\ R & - & \text { Radius of spring }(\mathrm{mm}) \\ D & - & \text { Diameter of spring }(\mathrm{mm}) \\ d & - & \text { Diameter of wire }(\mathrm{mm}) \\ \mathrm{r} & - & \text { Radius of wire }(\mathrm{mm}) \\ \tau & - & \text { Shear stress }(\mathrm{Mpa}) \\ \delta & - & \text { Deflection }(\mathrm{mm}) \\ c & - & \text { Modulus of rigidity }-80 \mathrm{Gpa} \\ n & - & \text { Number of turns of wire } \\ \mathrm{Mpa} & - & \text { Megapascal }\left(\mathrm{N} / \mathrm{mm}^{2}\right) \\ \text { Gpa } & - & \text { Gigapascal }\left(\mathrm{KN} / \mathrm{mm}^{2}\right)\end{array}$

\section{INTRODUCTION}

A suspension system is generally used for good handling performance and to provide isolation from high frequency vibrations emanating from the tires (the only major contact point) on uneven surfaces. They are employed to absorb shocks at the minimal cost of vehicles speed and thereby 
increase the comfort of the rider. This research is on the fluid damper systems, which is selfadaptive and is used on asphalt layouts to increase the speed and the acceleration of the two wheelers as compared to the front telescopic forks and rear spring shock absorber.

\section{LITERATURE REVIEW}

Ginder and Davis (1994) calculated the intermolecular forces and the shear stresses of the magneto - rheological fluid. They employed the finite element method to determine the average magnetic induction under non - linearity and saturation conditions. The shear stresses at low magnetic induction are controlled by the saturation and at high magnetic induction; the yield stress is calculation due to complete saturation. They employed Maxwell's stress tensor to find the average magnetic inductions, which were in agreement with previous magnetic fluids' induction value.

Cho, Jung and Lee (2005) have used a smart passive damper system with the aid of electromagnetic induction to effectively employ them in civil engineering applications such as in earthquake hazard mitigation control devices and in high-rise buildings. They used the magnetic energy created as a result of the interaction between the coil and permanent magnet to the passive damper and varied the damping characteristics of the passive damper system. Hence, they successfully employed the Newtonian First Law to the convert magnetic energy into mechanical energy to control the damper system.

Spelta, Savaresi and Fabbri (2010) have developed an algorithm of a control system for semi active damper system used in a two-wheeler motorcycle. The algorithm derived was then implemented in the Electronic Control Unit of the control system in the rear suspension of the motorcycle. The fabricated control system effectively proved the control system can be altered during motion.

\section{MAIN SECTION}

The current dampers that are in use are - magnetic dampers, hydraulic shock absorbers, position sensitive damping and spring shock absorbers.

Magnetic dampers use the repulsive properties of like poles of a magnet for riding on uneven or bumpy roads. However, this is limited to the building up magnetic strength and hence there is a time lag leading to break in contact between the wheel and the road and hence reducing the acceleration.

Hydraulic shock absorbers work on the movement of hydraulic fluid in the tubes converting shock energy into heat that has to be dissipated. Not all of the energy is converted and there are significant losses.

Position sensitive damping is smooth on the regular roads and has significantly less freedom in response to more irregular surfaces when the upward and downward movement of the piston starts to occur with greater intensity. The spring shock absorbers are limited to the material and the elasticity of the spring material.

\section{PROPOSED SOLUTION}

As stated in the beginning, a solution is found for the suspension system to be self-adaptive i.e. the suspension system adapts itself according to environmental factors influencing road 
conditions. So any uneven surface causes the damper to be operated with the help of a magnetic field. This varying position of the damper causes suspension to change from softer to harder for a certain rpm, in case of a bump or a pit (sudden impact). Generally, a wheel speed sensor is attached to the suspension system to adjudge when the damper system must be used according to our desired low and high rpm.

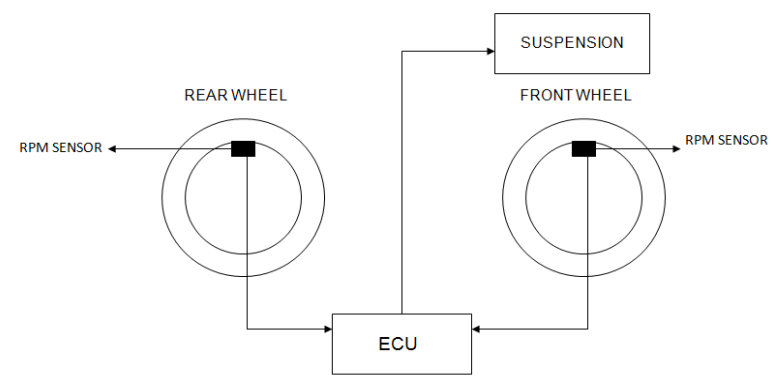

Figure 1. Sensors placed position in SFSAD

A wheel speed sensor monitors speed of wheel of vehicle and sends information to ECU, where it decides if the suspension is to be kept soft or hard. At high speeds, for immediate road contact the suspension is made hard by a switch controlled by ECU. At low speeds, the existing method is good enough to give comfort to the rider. The above principle works for both slow speeds and at high speeds and this self-adaptive damper is efficient at high speeds.

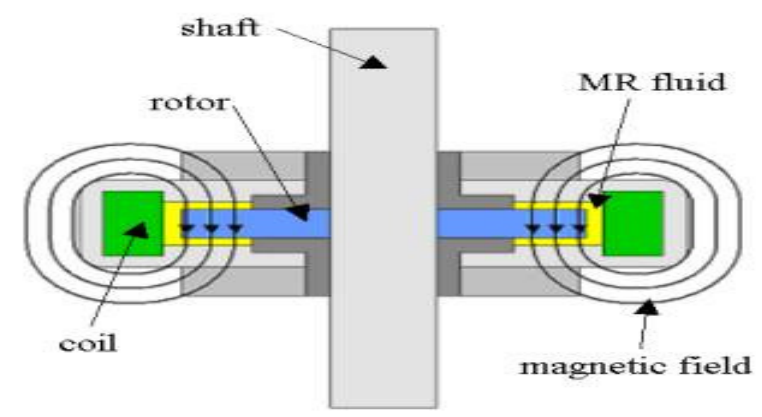

Lord MRB-2107-3

Figure 2. Pictorial representation of the working of the suspension system

The suspension system deployed on impact will activate the magnetic field. Figure 2 depicts the effect of the magnetic field around the fluid.The fluid particles get attracted due to the magnetic effect and they will align themselves to form a convulted and rigid pattern.

This damper system uses a "smart fluid" called the Magneto rheological Fluid. This fluid is the mainstay behind the working of our system. It employs the concept of dynamic viscosity that aligns the molecules in such a way that they resist motion. This resistance is actively used for absorbing the shocks and thereby not affecting the speed of the vehicle. 

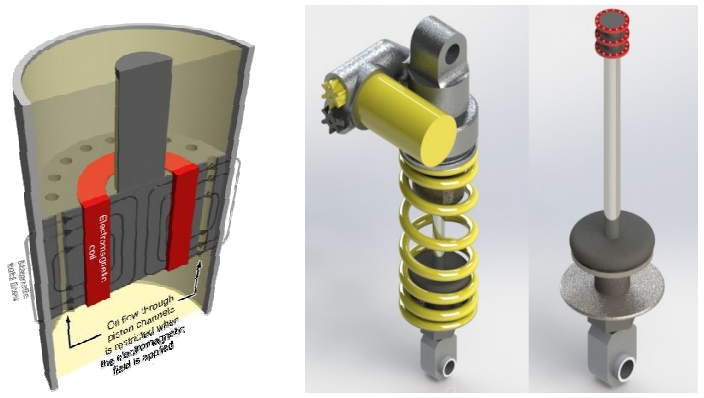

Figure 3. SFSAD 3D model designed in SolidWorks 2014

Ferrous particles are suspended in the hydraulic fluid, which changes when an external magnetic field is applied. When exposed to the magnetic field, the iron particles align themselves along the magnetic flux lines. They resist any flow or motion and act as a barrier to fluid flow. Change in yield stress is the reason for the change in viscosity, which is only an apparent change. Based on the Brownian movement, the surfactants get affected by the magnetic properties. MR(Magneto Rheological) fluids act as Bingham plastics i.e., they behave as rigid bodies at low stresses and viscous fluids at high stresses. Viscosity $(\eta)$ change requires little power.

Magneto rheological fluid generally consists of three major parts:

- Carbonyl iron particles - 3 to $5 \mu \mathrm{m}$ size, $30 \%$ of fluid

- Carrier liquid - Typical hydrocarbon oil, low viscosity

- Surfactants - Help to maintain particles in fluid

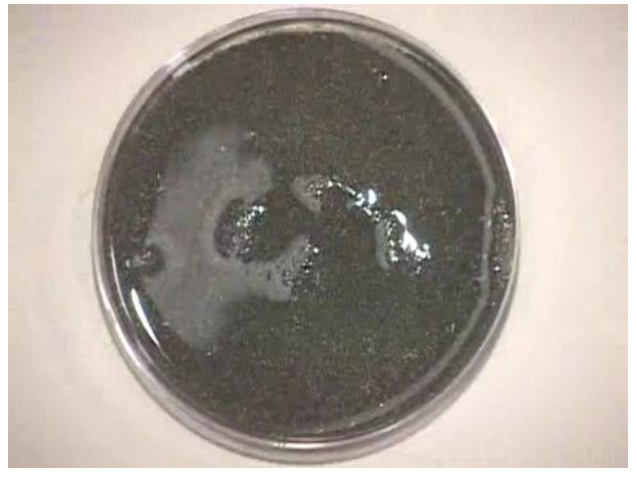

Figure 4. Depiction of a Magnetorheological fluid in real - life.

The damper is an electrically controlled viscous damper. MR fluid restricts fluid flow from one side of the piston to the other at "choking points". Piston is wound with magnetic coils, which is the source of magnetic field. Magnetic flux path is perpendicular to fluid flow and valve mode obtained. 


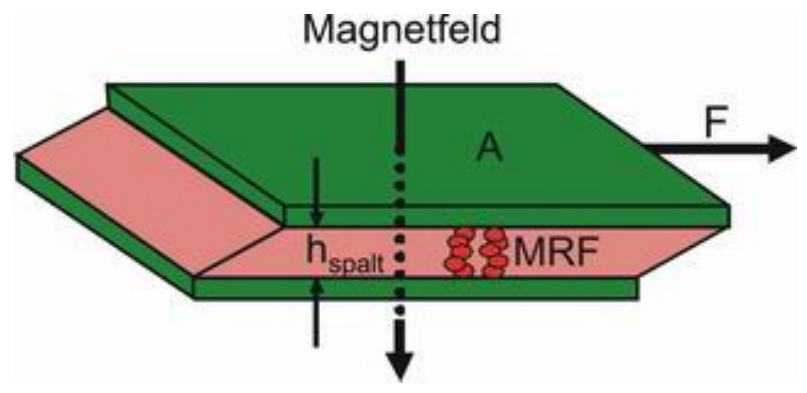

Figure 5. Two - Dimensional depiction of the effect of the magnetic field

Piston with annular holes moves along with the wheels. Suspension is soft i.e., fluid resistance is low. When the magnetic field is applied, the flow resistance increases and suspension becomes stiff thereby improving the handling to a great extent. Near-to-infinite settings are possible.

\section{DESIGNING PROCEDURES}

The designing of this damper was done using ANSYS and the calculations for designing components of the suspension are as follows.

To find diameter of the wire and spring, the following are assumed like, Load to be $150 \mathrm{~N}$, Coil diameter to be 8 times of wire, Shear stress to be $100 \mathrm{Mpa}$

$$
\begin{gathered}
W R=\frac{\pi}{\frac{15}{\pi}} \tau d^{3} \\
150 * 4 d=\frac{{ }^{3}}{16} * 100 * d^{3} \\
d=5.53 \mathrm{~mm} \cong 6 \mathrm{~mm} \\
E=8 d=8 * 6=48 \mathrm{~mm}
\end{gathered}
$$

To find deflection and shear stress, the following are assumed: Diameter of wire as $5 \mathrm{~mm}$, Number of turns as 12, Diameter of spring as $50 \mathrm{~mm}$, Axial load as $100 \mathrm{~N}$, Modulus of rigidity as $80 \mathrm{Gpa}$.

$$
\begin{gathered}
\delta=\frac{64 W^{3} n}{c d^{4}} \\
s=\frac{64 * 100 * 25^{3} * 12}{80 * 10^{2} * 5^{4}} \\
\delta=24 m m \\
W R=\frac{\pi}{15} \tau d^{3} \\
100 * 25=\frac{\frac{\pi}{N}}{16} * \tau * 125 \\
2500=24.54 \tau \\
\tau=101.9 \mathrm{MPa}
\end{gathered}
$$

Analysis was done in Ansys and the output was extracted in the form of a graph by knowing the values of stresses acting on every single point of the damper, thus generating the stress analysis from Ansys, which is as follows. 


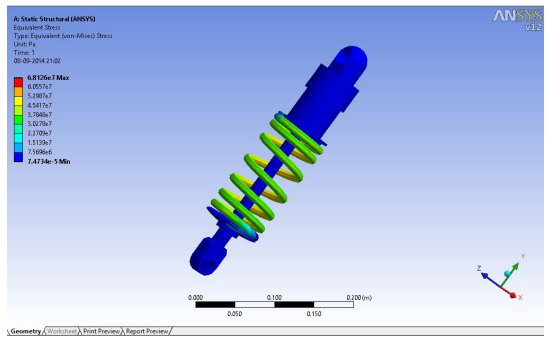

Figure 6. Stress analysis of SFSAD in Ansys 12.0 release

The graph output came as follows by using the simulated values from the Ansys by plotting it using Microsoft Excel. The graph is as follows.

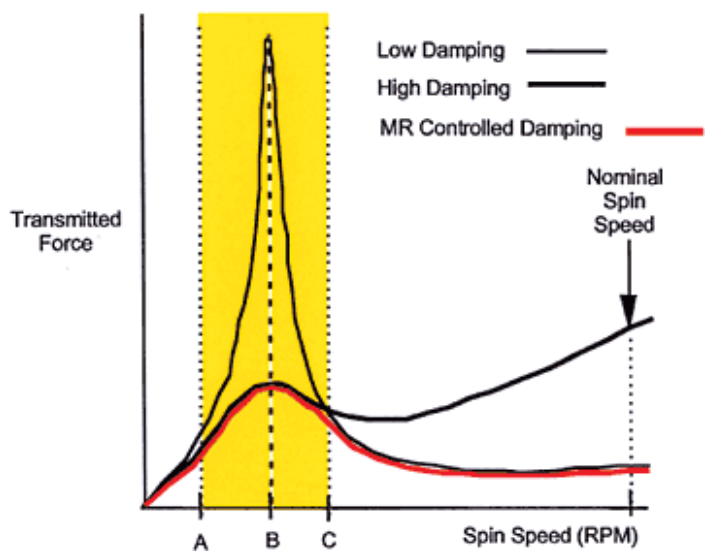

Figure 7. Effect of force is plotted for different speed conditions.

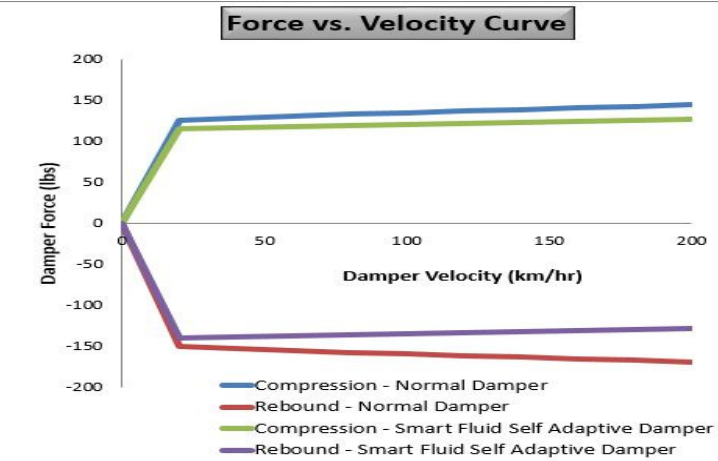

Figure 8. Damper Force vs Damper velocity curve plotted

\section{Advantages OF Proposed Solution}

Here are the advantages of our designed damper:

- Continuously varying damper settings - most effective balance obtained

- Very fast response time - very few milliseconds required for the change of settings

- Human intervention not needed but can be provided if required 
- Eliminates unreliable mechanical components - very high reliability

- Provides the best of both worlds - good riding performance and improved vehicle dynamics

- Applicable to all types of vehicles

- During the normal asphalt condition, it leads to more comfort and when in abnormal asphalt, this fluid increases the pace of the cars or motorcycles having this technology.

- This gives more acceleration and speed compromising a little on comfort.

\section{CONCLUSIONS}

Analysis on the Smart fluid self adaptive dampers in ANSYS shows that it gives good control of vehicle in bumps, more cornering stability and the ability to withstand lot of stresses acting on the tire patch during asphalt irregularities when compared to the conventional rear spring shock absorber and the front telescopic forks used predominantly. From the observations, it is evident that the system is highly reliable and can be implemented in automobiles.

\section{REFERENCES}

[1] http://www.nttyres.com/downloads/guide_to_dyno_graphs.pdf

[2] http://www.actawm.pb.edu.pl/volume/vol6no1/BAJKOWSKI_EN_2011_022.pdf

[3] http://people.duke.edu/ hpgavin/ermr/seattle01b.pdf

[4] http://www.lcool.org/technical/shock_absorbers/shock_absorbers_files/mono_tube.gif

[5] http://en.wikipedia.org/wiki/Shock_absorber

[6] http://img.youtube.com/vi/pRABTRAShzE/maxresdefault.jpg

[7] http://iopscience.iop.org/09641726/14/4/029

[8] http://www.sciencedirect.com/science/article/pii/S0967066110000432?np=y

[9] http://scitation.aip.org/content/aip/journal/apl/65/26/10.1063/1.112408 